\title{
Leidenschaften und Interessen: Hegel und die kritische Begründung der politischen Ökonomie Filipe Campello ${ }^{1}$
}

Zusammenfassung: Der vorliegende Aufsatz versucht die Hegelsche Theorie der Bürgerlichen Gesellschaft in einem aktualisierten Rahmen zu diskutieren und zwar insbesondere mit der Absicht, diesen in Bezug auf eine kapitalistisch geprägte und politisch-ökonomische Spannung zwischen Leidenschaften und Interessen darzustellen. Diese Argumente werden in zwei Stufen ausgeführt. In der ersten Stufe lässt sich Hegels Beitrag zu dieser Auffassung verstehen, als dass die Leidenschaft mit einem „bestimmten“ Gehalt verknüpft werden kann, welche sich aber erst durch einen spezifischen Bildungsprozess als „vernünftig“ begreifen lässt. Es wird somit bewiesen, dass Hegel diesen in dem Markt befindenden Selbstausdruck von Präferenzen und der Erfüllung von Selbstinteressen mit einer institutionellen vermittelte Willensbildung verbindet, wodurch die Handlungsmuster und individuelle Zwecke als einbeziehende Formen verstehen lassen. In der Zweiten Stufe wird verdeutlicht, dass die institutionellen Rahmen die Garantien einer wechselseitigen Befriedigung individueller Interessen fördern müssen, die aber den im Markt und auf der Arbeit handelnden Akteuren nicht extern bleibt, sondern sich auf ein legitimes soziales Teilnahmegefühl gründet. Es wird zuletzt eine ,institutionalisierte Solidarität“ vorgeschlagen, die der Zufälligkeit von Gefühle komplementär ist: Diese Sphären sind normativ legitimiert, wenn diese - nach dem Muster des freien Willens - die wechselseitige Anerkennung und Befriedigung individueller Präferenzen einerseits und die an sozialen Verhältnissen dezentrierte Interessen andererseits ausdrückt.

Schlüsselwörter: Gefühle, Institutionen, soziale Gerechtigkeit, institutionalisierte Solidarität, Markt, Freier Wille, soziale Freiheit.

Resumo: O presente artigo discute a teoria hegeliana da sociedade civil dentro de um quadro atual, tendo em vista principalmente a tensão entre paixões e interesses encontrada no modelo econômico-político do capitalismo. Essa discussão será desenvolvida em dois momentos. Primeiramente, apresento a contribuição teórica de Hegel a esse debate a partir da concepção de que as paixões se ligam a um conteído "particular", que somente são concebidas como "racionais" através de um processo de formação especifico. Eu procuro mostrar que Hegel liga essa expressão individual de preferências e o preenchimento de interesses pessoais através de uma formação da vontade institucionalmente mediada, a partir da qual os padrões de ação e de fins individuais são compreendidos enquanto formas inclusivas. Em um segundo momento, proponho que um quadro institucional deve promover as garantias de uma satisfação reciproca de interesses individuais, que, ao mesmo tempo, não sejam concebidos externamente aos participantes no mercado e no trabalho, mas que sejam fundamentadas a partir de uma legítimo sentimento de pertencimento social. Concluo com a proposta de uma "solidariedade institucionalizada", que deve ser complementar à contingência do sentimento individual: essas esferas só são legitimadas normativamente quando elas - segundo o padrão da vontade livre - expressa, por um lado, o reconbecimento reciproco e a satisfação de preferências individuais, e, por outro, interesses descentrados em vista das relações sociais.

Palavras-chaves: Emocões, instituições, justiça social, solidariedade institucionalizada, mercado, vontade livre, liberdade social.

\footnotetext{
${ }^{1}$ Doutor pela Goethe-Universität Frankfurt; pós-doutorado pela Universidade Federal de Pernambuco. Email: filipebcampello@gmail.com
} 
Filipe Campello

In seiner klassischen Studie Leidenschaften und Interessen rekonstruiert Albert Hirschman mit einer reichen ideengeschichtlichen Untersuchung die im Diskurs der Moderne geschehenden begrifflichen Veränderungen, wobei das ,Interesse“ das Grundmotiv für die Legitimierung der Entstehung des Kapitalismus bildet ${ }^{2}$. Hirschman zeigt, wie der Anfang des kapitalistischen Diskurses nicht durch Unterdrückung von Leidenschaften charakterisiert wurde, sondern vielmehr durch das „Prinzip der wechselseitigen Neutralisierung der Leidenschaften“33. Nach diesem Prinzip - welche die Tradition von Machiavelli über Bacon, Spinoza bis Hume geprägt hat - sind die Leidenschaften nur mit anderen Leidenschaften $\mathrm{zu}$ konfrontieren ${ }^{4}$. Insofern ist die Grundidee Hirschmans Untersuchung, dass das Interesse nicht eine den Leidenschaften gegenüber stehende „vernünftige“, kalkulierende Dimension bezeichnet, sondern vielmehr eine stets von Leidenschaften geprägte Struktur, wodurch die oft beschriebene Dichotomie zwischen Leidenschaften und Interesse in Frage gestellt wird. Dadurch will Hirschman zeigen, dass die Entstehung des Interesses als Grundprinzip der Ökonomie von einer Leidenschaftsdimension geprägt war, die in der Lage war, dem entstehenden Kapitalismus eine legitimierende Basis zu verschaffen. Die Idee im Hintergrund besteht darin, dass es legitim wäre, denjenigen

\footnotetext{
${ }^{2}$ HIRSCHMAN 1987.

${ }^{3}$ EBD., S. $28 \mathrm{ff}$.

${ }^{4}$ EBD., S. 24 ff. So etwa zitiert Hirschman Bacons Vorstellung, „to set affection against affection and to master one by another" (BACON, The advancement of Learning); Spinozas Ethik: "An affect cannot be restrained nor removed unless by an opposed and stronger affect" (Part IV, propos. 7) und auch „No affect can be restrained by the true knowledge of good and evil insofar as it is true, but only insofar as it is considered as an affect" (Part IV, propos. 14) und Hume: „Nothing can oppose or retard the impulse of passion but a contrary impulse.“ (Treatise, book III, Part II, Section II)
}

\section{Revista de Filosofia Moderna e Contemporânea Brasília, no 2, ano 1, 2013.}




\section{Leidenschaften und Interessen: Hegel und die kritische Begründung der} politischen Ökonomie

Leidenschaften, die als negativ angesehen werden, die Leidenschaft der Lust auf Geld gegenüberzusetzen. Das mit dem Gelderwerb verbundene ,Interesse“ wurde daher als eine positive, „ruhige Leidenschaft ${ }^{\star 5}$ in einem legitimierenden Diskurs des Kapitalismus eingebracht, insofern dieses 'Interesse' gegenüber anderen Leidenschaften den Vorteil hätte, voraussehbar und beständig zu sein, was für die entstehende Markttauschen wichtig wäre ${ }^{6}$ und damit als eine motivierende und moralisch legitimierte Kraft für das Streben nach Kapitalakkumulation diente.

Mit dieser Interpretation wird gezeigt, dass die Idee von individueller Befriedigung selbst in einer schwierigen Spannung zu einem,vernünftigen Zweck steht, insofern die zu befriedigenden Bedürfnisse von vielfältigen und auch gewiss, vorrationellen' Aspekten geprägt sind. Und zudem lässt sich die auf den Markt beziehende strategische Rationalität von einem emotionalen und sozial vermittelten Gehalt nicht trennen ${ }^{7}$. Nach dieser Auffassung werden die Interessen auf einem Paradigma basiert und dadurch legitimiert, dass Profit und individuelle Befriedigung assoziiert werden - und dieser daher eine emotionellen Kraft besitzt, so dass diese Rechtfertigung nicht nur durch rationelle, sondern auch emotive Aspekte aufweist. Die Fragilität der mit dem rational choice Ansatz verbundenen Interpretationen besteht vor allem in ihrem begrenzten Erklärungspotenzial (etwa in Bezug auf reale Motivationen zum Profit und ihrer Begründungen), ihrer Unfähigkeit also, eine vorherige

\footnotetext{
${ }^{5}$ EBD., $72 \mathrm{ff}$.

${ }^{6}$ EBD., 57 ff. Vgl. auch Gunn 1968.

${ }^{7}$ Die strategische Rolle der Gefühle für die ökonomischen Handlungen diskutiert FRANK 1998.
} 
Filipe Campello

Grunddifferenzierung von denjenigen Faktoren darzustellen, die dahin führt, den Profit als Ziel zu setzen, wobei die Rolle der Leidenschaft von Gelderwerb nicht nur als ein Mittel, sondern auch ein Zweck an sich angesehen wird.

Dieses vereinfachte Bild eines viel komplexeren Problems soll vor allem zeigen, dass die ökonomische Bedeutung von Interesse sich nur in Hinblick auf wechselseitige soziale Legitimierung (,social space of reasons') einerseits und andererseits in Hinblick auf ihre leidenschaftliche Dimension erklären lässt. Die falsche Opposition zwischen Vernunft und Gefühl ist sowohl eine angebliche Rechtfertigung dafür, das Interesse als einen vernünftigen Grund gegen die Leidenschaften zu setzen, als auch ein Hindernis dafür, die vernünftigen Gründe für das Streben nach Profit als begrenzende Erklärung anzuerkennen, wodurch eine grundlegende leidenschaftliche Komponente vernachlässigt wird.

Mit diesem Bild vor Augen lässt sich ein zweites Problem zeigen: die interne begriffliche Veränderung zwischen Leidenschaften und Interesse bezeichnet schon im Anfang nicht nur die Legitimierung von Gelderwerb, sondern wurde auch mit isolierten selbstsüchtigen Interessen ausgeglichen: während sich einerseits die Annahme des Interesses als etwas untrennbar mit Leidenschaften verbundenen stützen lässt, so dass die Interessen selbst nicht nur als rationelle kalkulierende Struktur, sondern als eine Umformung von Leidenschaften zu gelten haben, bleibt nun andererseits unklar, wie ein auf den Markt bezogenes Interesse durch die Kriterien wechselseitiger Befriedigungen übersetzt werden kann. Zusammen mit dem Argument einer 


\section{Leidenschaften und Interessen: Hegel und die kritische Begründung der}

politischen Ökonomie

nicht bloß rationellen strategischen Handlung bleibt noch in einem zweiten Schritt einer Willensbildung aufzuzeigen; nicht nur zeigt das Interesse ein leidenschaftliches Motiv, das von der Legitimierung kapitalistischen Handelns verkörpert wird, sondern es braucht in einem zweiten Schritt eine Ausbildung von denjenigen Interessen, die in einem ersten Moment nur als Sucht nach Befriedigung von individuellen Bedürfnissen gelten könnten. Der Bildungsprozess der Interessen andererseits besteht darin - folgen wir Hirschmans Untersuchung -, die ökonomische Praxis und auf den Markt bezogene Interessen nicht nur als strategische Kalkulation von Gründen und Zielen zu verstehen, sondern auch als unerlässliche Formierung auf der Ebene der Bedürfnisse und Leidenschaften. Wenn diese Dichotomie aufgelöst wird, ist noch $\mathrm{zu}$ erklären, wie diese Leidenschaften als „kooperierende“ herausgebildet werden kann.

An keiner Stelle aber der kapitalistischen Diskurse ist nun zu verstehen, wie die Interessen sich auf kooperierende oder einbeziehende Leidenschaft beziehen könnten. Auch wenn sich diese Motive als zentral für das ökonomische Handeln zeigen, bleiben sie undeutlich, wenn sie nicht durch eine vorherige Fragestellung über die Entstehung der Interessen erklärt werden. Ich werde im Folgenden die Interpretation vorschlagen, dass in Hinblick auf Hegels Rechtsphilosophie ein spezifischer Bildungsprozess innerhalb der Sphäre der Wirtschaftsverhältnisse nötig wäre, damit die derartigen Interessen auch einen Ausdruck des sittlich vermittelten ,freien Willens` bezeichnen könnten, von dessen Erläuterung die Vorstellung entsteht, dass die Interessen Anderer beachtet werden müssen. Die für uns 
Filipe Campello

grundlegende Relevanz Hegels Ansatzes ist derart, dass sich zwar eine zentrale Rolle von subjektiven Interessen ergibt, die aber nicht nur in der Befriedigung selbstzentrierter Interessen liegt, sondern in dem Gefühl wechselseitiger Abhängigkeit zwischen meiner Befriedigung und der Befriedigung Anderer. Und insofern setzt die Auffassung ökonomischer Rationalität die Erläuterung der sozialen Bedeutung der Sittlichkeit voraus ${ }^{8}$ : Es ist als Verwirklichung sittlicher Freiheit mit der die Selbstinteressen als „vernünftig“ beschrieben werden.

Wie diese Interpretation vorschlagen möchte, erfolgt in den Ökonomischen Verhältnissen eine spezifische Bildungsform, deren jeweils geforderte Bedürftigkeit erst durch Sozialisationsprozesse zu erfüllen sind. Daher lässt sich das Interesse nicht nur als ein anderer Begriff für Bedürfnis auffassen, sondern als ein geformtes Bedürfnis, nämlich ein auf den Markt bezogenes? ${ }^{9}$ Dies ist der nach Hegels Theorie der bürgerlichen Gesellschaft durchgeführte Lernprozess, hinter welchem die Idee steht, die Hirschman formuliert: Leidenschaften müssen durch den Sozialisationsprozess in Interessen umformuliert werden, die sich in nüchterner, kalkulatorischer Form auf dem Markt einfinden können. Insofern lässt sich Hegels Beitrag zur Auffassung von Leidenschaft gegen Leidenschaft in der Konzeption sehen,

\footnotetext{
${ }^{8}$ Vgl. WALTON (1984). Der Autor behauptet, dass das Argument der Verwirklichung subjektiver Freiheit in der sittliche Sphären "[has] important implications for Hegel's theory of rational economic activity. The application of empirical and analytical criteria in respect of the determination of means to ends is rejected as sufficient condition of rationality. Economic rationality is, rather, dependent upon the clarification of the norms and values of ethical life. A coherent ethical life is thus condition of economic rationality" (250).

${ }^{9}$ HONNETH (2001) sieht „Interesse“ als einen besseren Begriff dafür an, was Hegel in der bürgerlichen Gesellschaft als Bedürfnis darstellt.
} 
Leidenschaften und Interessen: Hegel und die kritische Begründung der politischen Ökonomie

dass die Leidenschaft mit einem ,bestimmten' Gehalt verknüpft werden kann, welcher sich erst durch eine Ausbildung als „vernünftig“ begreifen lässt. Es ist nach Hegel nicht eine Leidenschaft, die sich über eine andere erhebt, sondern eine auf dem Markt in Form von Interesse angenommene Leidenschaft, die einen spezifischen Bildungsprozess erfährt.

\section{Zur Herausbildung kooperierender Interessen}

Verschiedene Interpretationen der Verortung der Ökonomie in Hegels System sehen sich als erstes mit der Schwierigkeit konfrontiert, Hegels Konzeption der bürgerlichen Gesellschaft zu kontextualisieren ${ }^{10}$. Gewiss entspricht die aktuelle Debatte über die bürgerliche Gesellschaft nicht grundsätzlich einer auf den Markt bezogenen, die eine Anpassung an Hegels ursprüngliche Auffassung erfordert. In diesem Sinn entstehen zwei Grundlinien in Hinblick auf Hegels Theorie der bürgerlichen Gesellschaft: Während Hegel einerseits die Auffassung von Öffentlichkeit, demokratischer Willensbildung und den Raum von Konflikten und öffentlicher Debatte in der Sphäre des Staates verortet hat, finden Hegel zufolge die ökonomischen

${ }^{10}$ Für hilfreiche Gesamtdarstellungen von Hegels Theorie der Ökonomie vgl. unter anderen: CHAMLEY 1963, 1965; AVINERI 1972; RIEDEL 1970; COHEN/ARATO (1992) haben Hegel als einen bahnbrechenden Denker über die bürgerliche Gesellschaft dargestellt, insofern er diese nicht nur als von selbstinteressierten Verhältnissen geprägte Sphäre gesehen hat, sondern auch als eine von moralischen Verhältnissen geprägte Sphäre von Solidarität und Wohlstand. Vgl COHEN/ARATO 1992. 
Filipe Campello

Verhältnisse in der Sphäre der bürgerlichen Gesellschaft statt, die sich später aber durch neue Konfigurationen ausdifferenziert ${ }^{11}$.

Das ist aber symptomatisch und besitzt aktuelle Relevanz, dass Hegel die bürgerliche Gesellschaft und den Markt nicht entkoppelt hat. Es war ihm wohl vor Augen, dass der Markt nicht als eine autonome Sphäre zu verstehen ist, sondern innerhalb der sittlichen Beziehungen steht und als neue Stufe des Individualisierungsprozesses begriffen werden kann; denn auch hinsichtlich der Marktverhältnisse sollen die Akteure lernen, wie sie von einem willkürlichen zu einem kooperierenden Willen gelangen. Nur insofern der Markt innerhalb der bürgerlichen Gesellschaft verortet wird - wenn wir Hegels Argument folgen - kann er seine spezifische Bildungsform erhalten.

Insbesondere mit der Lektüre von Adam Smith erfasst Hegel die bürgerliche Gesellschaft als eine notwendige Sphäre der Individualisierung, die neue Formen von Bedürfnisbefriedigungen ermöglicht: In der bürgerlichen Gesellschaft sind die Individuen durch „das Band des gegenseitigen Bedürfnisses“ $(\$ 33, Z \text { Z. })^{12}$ verbunden, dessen Befriedigung nur sozial vermittelt ermöglicht wird. Hegel war sich dessen überzeugt - mit dem Hinweis auf „Smith, Say and Ricardo“ (\$189) - dass die Realisierung allgemeiner Befriedigung durch Selbstinteresse gelten sollte. Daher fand der deutscher

11 In dieser Richtung entwickelt Axel Honneth noch in Kampf um Anerkennung die Aktualisierung der Sphäre der bürgerlichen Gesellschaft, während in seinem letzten Buch Das Recht der Freiheit diese Sphäre als ,Markt" entwickelt wird. Unter der umfassenden Literatur zum gesamten Überblick über Hegels Theorie der bürgerlichen Gesellschaft nenne ich als besonders instruktiv für meine Auffassung: die gesammelten Bände Pelczynski (1984), insb. WALTON (1984); HORSTMANN 1997; RIEDEL (Hg.) 1975.

${ }^{12}$ Wenn nicht anders angegeben, zitiere ich Hegels Rechtsphilosophie nach den Werken in 20 Bänden (Hrsg. von Eva Moldenhauer und Karl Markus Michel (1970), Frankfurt a. M.: Suhrkamp, Band VII) durch die Angabe von Paragraph.

\section{Revista de Filosofia Moderna e Contemporânea}

Brasília, no 2, ano 1, 2013. 


\section{Leidenschaften und Interessen: Hegel und die kritische Begründung der} politischen Ökonomie

Philosoph die Studien der ehemaligen britischen ,Staatsökonomie' fruchtbar, insofern sie anstrebten, „zu einer Masse von Zufälligkeiten die Gesetze [zu] finde[n]“ (\$189). Mit der britischen Ökonomie kommt Hegel gleichzeitig zur Behauptung, dass ein „Allgemeines“ die Voraussetzung für die Verwirklichung von privaten Interessen und Zwecken ist, so dass in der bürgerlichen Gesellschaft die Verhältnisse zwischen privaten Zwecke zwar vorkommt, welche aber „durch das Allgemeine vermittelt“ (\$187) sind. Es ist allerdings kontrovers, inwiefern Hegel mit Adam Smith die Auffassung eines eher optimistischen Glaubens an den organischen Charakter des Marktes teilt, durch welchen die Befriedigung von Selbstinteresse zu einer allgemeinen Befriedigung führt ${ }^{13}$. Mit seiner These der Vernünftigkeit war Hegel zwar überzeugt - und daher tendenziell mit dem freien Markt und der „invisible hand" übereinstimmend -, dass in der bürgerlichen Gesellschaft die Suche nach Erfüllung von Selbstinteressen zur Befriedigungen des Allgemeinen führt ${ }^{14}$; andererseits aber lässt sich von Hegels Prämissen ausgehend auch behaupten, dass nur von der These der Realisierung von Selbstinteresse ausgehend es zur Destabilisierung wechselseitiger Befriedung führen könnte,

\footnotetext{
${ }^{13}$ Vgl. zur Diskussion HERZOG 2013, HONNETH 2011, RIEDEL (Hg.) 1975.

${ }^{14}$ Auch wenn Hegel sich strukturell gesehen in Richtung einer stärker regulierenden Rolle des Staates orientiert. Zudem weist Hegel auf eine notwendige Regulierung nicht nur durch den Staat hin, sondern betont die sich innerhalb der bürgerlichen Gesellschaft befindenden Mechanismen, wie etwa die Hegels Auffassung entsprechenden spezifischen Bedeutungen von ,Rechtspflege' und ,Polizei‘. Die umfassende Diskussion zum Verhältnis zwischen Staat und Markt einerseits und den mit der bürgerlichen Gesellschaft verbundenen, regulatorischen Institutionen andererseits werden wir hier nicht vertiefen, da es unser hier verfolgtes Ziel eher ist, den innerhalb der bürgerlichen Gesellschaft selbst durchzuführenden Lernprozess zu betonen. Zum Anschluss an Hegels Debatte vgl. RIEDEL 1970, 1975.
} 
Filipe Campello

wenn die Teilnahme an der sozial vermittelten Sphäre der Arbeit und des Marktes von dem Modell des „freien Willens“ abweicht.

In seiner Auffassung artikuliert Hegel eine doppelseitige Bedeutung von subjektiven Pflichten gegenüber der bürgerlichen Gesellschaft und den durch sie ermöglichten individuellen Rechtsschutz („Die bürgerliche Gesellschaft muß ihr Mitglied schützen, seine Rechte verteidigen, so wie der Einzelne den Rechten der bürgerlichen Gesellschaft verpflichtet ist“" (\$238, Z.). Mit dieser besonders Art von sozialem Boden und dem Schutz der Individualität verkörpert die bürgerliche Gesellschaft die primäre nur durch die Familie charakterisierte Erziehungsrolle. Weil aber die Familie nicht in der Lage ist, Bedürfnisse jenseits der natürlichen (wie Liebe und Affekte) zu befriedigen, sieht Hegel in der bürgerlichen Gesellschaft zwar auch als ein „System der Bedürfnisse“, jetzt aber nicht mehr der natürlichen, sondern pluralen Bedürfnisse, die innerhalb der Gesellschaft wechselseitig zu befriedigen sind. Mit der Auffassung eines „Systems der Bedürfnisse“ zeigt Hegel, dass es in der bürgerlichen Gesellschaft um einen anderen Typus von individuellen Bedürfnissen geht, die nur durch Arbeitsteilung und Marktverhältnisse befriedigt werden können. Wenn die Familie die Befriedigung von ,unmittelbaren', von Natur geprägten Bedürfnisse ermöglicht,

[reißt] die bürgerliche Gesellschaft [...] das Individuum aus diesem Bande heraus, entfremdet dessen Glieder einander und anerkennt sie als selbständige Personen; sie substituiert ferner statt der äußeren unorganischen Natur und des väterlichen Bodens, in welchem der Einzelne seine Subsistenz hatte, den ihrigen und unterwirft das Bestehen der ganzen Familie selbst, der Abhängigkeit von ihr, der Zufälligkeit. So ist das 


\section{Leidenschaften und Interessen: Hegel und die kritische Begründung der politischen Ökonomie}

Individuum Sohn der bürgerlichen Gesellschaft geworden, die ebensosehr Ansprüche an ihn, als er Rechte auf sie hat. (\$238).

Die Bürger können sich dadurch als autonome Personen fühlen, die eine Vielfalt von Bedürfnissen erfahren. Das Subjekt, das in der Familie einen noch teilweise natürliche, selbstzentrierte Wille ausdrückt, erfährt darum in der bürgerlichen Gesellschaft eine erweitere und notwendige Stufe für die Realisierung des „freien Willens“, das sich nur innerhalb des erweiterten Verhältnisses als „frei“ realisieren lässt - also weder einen negativen noch einen ,zufälligen` und begrenzenden Inhalt hat. Nach Hegel lässt sich in der bürgerlichen Gesellschaft kein Abbruch der Konstitution des freien Willens sehen, sondern vielmehr eine gewichtige Sphäre der Formierung praktischer Individualität, die mit einer aktiven Form von Teilnahme verbunden ist, in welcher sich subjektive Interessen als ständige Expression der Individualität auf einer erweiterten Stufe verwirklichen lassen. ${ }^{15}$ In diesem Sinne hat Hegel die bürgerliche Gesellschaft als den Platz begriffen, an dem „das Prinzip der selbständigen in sich unendlichen Persönlichkeit des Einzelnen, der subjektiven Freiheit“ (\$185) sich ausdrückt. Es ist aber nur dadurch zu behaupten, dass das Subjekt auch eine legitime Expression einer einbeziehenden Perspektive verwirklicht, wobei der auf den Markt bezogene Wille als „frei“" verstanden wird. ${ }^{16}$

15 Insofern findet in der bürgerlichen Gesellschaft der Prozess statt, „die Einzelheit und Natürlichkeit derselben durch die Naturnotwendigkeit ebenso als durch die Willkür der Bedürfnisse zur formellen Freiheit und formellen Allgemeinheit des Wissens und Wollens zu erheben, die Subjektivität in ihrer Besonderheit zu bilden.“( $(187)$.

${ }^{16}$ Für den Markt als Sphären der Verwirklichung der „sozialen Freiheit“ vgl. HONNETH 2001, Teil C, Kap. III.2 
Filipe Campello

Mit dieser Prämisse lässt sich zum folgenden Modell fortschreiten: Das Subjekt erreicht seine Befriedigung in der bürgerlichen Gesellschaft insofern es einerseits in seiner Tätigkeit sich selbst erkennen lässt und andererseits seine Interessen verwirklichen kann, welche aber - im Unterschied zu einem utilitaristischen Ansatz - innerhalb der Struktur des „freien Willens“ und seinem Bildungsprozess auszudrücken sind. Insofern sieht Hegel, dass die Bürger im „gesellschaftlichen Bedürfnisse“ - im Unterschied zur „Naturnotwendigkeit des Bedürfnisses“ - vielmehr eine neue Stufe einer „Befreiung“ (\$194) erfahren.

Insofern erfasst Hegel die „Sphäre“ der „Wechselbeziehung der Bedürfnisse und der Arbeit“ als einen Prozess der „Bildung“ (\$209). ${ }^{17}$ Mit der durch Arbeit und Markt ermöglichten Bildung entsteht das, was Hegel „Hemmung der Begierde“ nennt, insofern in der bürgerlichen Gesellschaft nicht bloß eine Begierde befriedigt wird ${ }^{18}$. In dieser Richtung erfasst Hegel den sich hier vollziehenden Bildungsprozess wie folgt:

Die Bildung ist daher in ihrer absoluten Bestimmung die Befreiung und die Arbeit der höheren Befreiung, nämlich der absolute Durchgangspunkt zu der nicht mehr unmittelbaren, natürlichen, sondern geistigen, ebenso zur Gestalt der Allgemeinheit erhobenen unendlich subjektiven Substantialität

\footnotetext{
17 „Das Relative der Wechselbeziehung der Bedürfnisse und der Arbeit für sie hat zunächst seine Reflexion in sich, überhaupt in der unendlichen Persönlichkeit, dem (abstrakten) Rechte. Es ist aber diese Sphäre des Relativen, als Bildung, selbst, welche dem Rechte das Dasein gibt, als allgemein Anerkanntes, Gewußtes und Gewolltes zu sein und, vermittelt durch dies Gewußtund Gewolltsein, Gelten und objektive Wirklichkeit zu haben“. (\$209)

18 „In der Vervielfältigung der Bedürfnisse liegt gerade eine Hemmung der Begierde, denn wenn die Menschen vieles gebrauchen, ist der Drang nach einem, dessen sie bedürftig wären, nicht so stark, und es ist ein Zeichen, daß die Not überhaupt nicht so gewaltig ist.“ (§191, Z.).
}

\section{Revista de Filosofia Moderna e Contemporânea$$
\text { Brasília, no 2, ano 1, } 2013 .
$$ 


\section{Leidenschaften und Interessen: Hegel und die kritische Begründung der}

politischen Ökonomie

der Sittlichkeit. Diese Befreiung ist im Subjekt die harte Arbeit gegen die bloße Subjektivität des Benehmens, gegen die Unmittelbarkeit der Begierde sowie gegen die subjektive Eitelkeit der Empfindung und die Willkür des Beliebens. Daß sie diese harte Arbeit ist, macht einen Teil der Ungunst aus, der auf sie fällt. Durch diese Arbeit der Bildung ist es aber, daß der subjektive Wille selbst in sich die Objektivität gewinnt, in der er seinerseits allein würdig und fähig ist, die Wirklichkeit der Idee zu sein. (\$187)

Zur Bildungsrolle in der bürgerlichen Gesellschaft lassen sich bei Hegel grundsätzlich zwei Prinzipien hinsichtlich der Auffassung der Person finden: Sie besteht einerseits als „Vermischung von Naturnotwendigkeit und Willkür“ und andererseits als „Beriehung auf andere“ (\$182), insofern sich seine Bedürfnisse nur durch Anderen befriedigen lassen. Die Bildungsfunktion besteht schon in der wechselseitigen Abhängigkeit zur Befriedigung von individuellen Bedürfnissen. ${ }^{19}$ Und hier - schreibt Hegel weiter - ist es, wo „die Wellen aller Leidenschaften ausströmen“ (182, Z.). In diese Vermittlung durch Andere wird jeder in seiner Selbstbefriedigung durch intersubjektive Verhältnisse geformt ${ }^{20}$ in welcher - wie in Smiths Auffassung - sich selbstsüchtige Zwecke auf ein Allgemeines beziehen:

Der selbstsüchtige Zweck in seiner Verwirklichung, so durch die Allgemeinheit bedingt, begründet ein System allseitiger Abhängigkeit, daß die Subsistenz und das Wohl des Einzelnen und sein rechtliches Dasein in die Subsistenz, das Wohl und

19 „In der bürgerlichen Gesellschaft ist jeder sich Zweck, alles andere ist ihm nichts. Aber ohne Beziehung auf andere kann er den Umfang seiner Zwecke nicht erreichen“ (§182, Z.).

${ }^{20}$ In dieser Richtung schreibt Habermas: "Sittlich nennt Hegel einen gesellschaftlichen Zustand, in dem alle Glieder zu ihrem Recht kommen und ihre Bedürfnisse befriedigen" (1966:355). 
Filipe Campello

Recht aller verflochten, darauf gegründet und nur in diesem Zusammenhange wirklich und gesichert ist . $(\$ 183)^{21}$

Diesen Bildungsprozess sieht Hegel als Ort der Vermittlung zwischen individuellem Interesse und Einbeziehung der Interesse Anderer besonders in den „Korporationen“ und „Ständen“. Auch wenn diese Begriffe inzwischen obsolet wurden, bleiben Hegels Intuitionen hinsichtlich der Korporation und dem Gehalt eines Allgemeinen unter der Pluralität und Zufälligkeit der besonderen Interesse, welche konstitutiv für die bürgerliche Gesellschaft ${ }^{22}$ sind, noch relevant: Durch die sozialen Dimension der Befriedigung subjektiver Bedürfnisse (\$192), die wechselseitige Arbeitsteilung (\$198) und die Aufteilung des Marktes in Stände werden die Tätigkeiten von Anderen zur Bedingung für die individuellen Befriedigungen (\$201). So etwa in der

Abhängigkeit und Gegenseitigkeit der Arbeit und der Befriedigung der Bedürfnisse schlägt die subjektive Selbstsucht in den Beitrag zur Befriedigung der Bedürfnisse aller anderen um, in die Vermittlung des Besonderen durch das Allgemeine als dialektische Bewegung, so daß, indem jeder für sich erwirbt, produziert und genießt, er eben damit für den Genuß der Übrigen produziert und erwirbt. (\$199)

\footnotetext{
${ }^{21}$ Und an einer anderen Stelle begreift Hegel die hier sich befindende Bedeutung des „Allgemeinenheit" durch den Prozess, „de[n] Anderen [zu] richten“ und ,ihre Meinung an[zu]nehmen": Dadurch, daß ich mich nach dem anderen richten muß, kommt hier die Form der Allgemeinheit herein. Ich erwerbe von anderen die Mittel der Befriedigung und muß demnach ihre Meinung annehmen. Zugleich aber bin ich genötigt, Mittel für die Befriedigung anderer hervorzubringen. Das eine also spielt in das andere und hängt damit zusammen. Alles Partikulare wird insofern ein Gesellschaftliches $(\$ 192, Z$.)

22 „Indem nach der Idee die Besonderheit selbst dieses Allgemeine, das in ihren immanenten Interessen ist, zum Zweck und Gegenstand ihres Willens und ihrer Tätigkeit macht, so kehrt das Sittliche als ein Immanentes in die bürgerliche Gesellschaft zurück; dies macht die Bestimmung der Korporation aus“ (\$249).
}

\section{Revista de Filosofia Moderna e Contemporânea}

Brasília, no 2, ano 1, 2013. 


\section{Leidenschaften und Interessen: Hegel und die kritische Begründung der}

politischen Ökonomie

In den Korporationen finden die Individuen ihre Individualität und Fähigkeiten „anerkannt“ (\$253): „So ist auch anerkannt, daß es [,das Mitglied einer Korporation“ (F.C.)] einem Ganzen, das selbst ein Glied der allgemeinen Gesellschaft ist, angehört und für den uneigennützigeren Zweck dieses Ganzen Interesse und Bemühungen hat; - es hat so in seinem Stande seine Ehre“ (\$253). Und nur bei der Korporationen - insofern „gesetzliche konstituiert und anerkannt", erhebt einerseits die Arbeit einen „sittlichen Grund" und andererseits werden Probleme der wirtschaftliche Sphäre der bürgerlichen Gesellschaften minimisiert:

In der Korporation verliert die Hilfe, welche die Armut empfängt, ihr Zufälliges sowie ihr mit Unrecht Demütigendes und der Reichtum in seiner Pflicht gegen seine Genossenschaft den Hochmut und den Neid, den er, und zwar jenen in seinem Besitzer, diesen in den anderen erregen kann; - die Rechtschaffenheit erhält ihre wahrhafte Anerkennung und Ehre. (\$253)

In der Verwicklung zwischen „Rechtschaffenheit“, „Ehre“ und Anerkennung" lassen sich die Begriffe, die Hegel in der bürgerliche Gesellschaft als Voraussetzung für eine neue Stufe der Individualisierung ansieht, artikulieren. Die durch die Teilnahme an den Ständen und Korporationen durchgeführte Herausbildung des Selbstgefühl als anerkannt und geehrt ermöglicht eine Reorientierung der selbstsüchtigen Werte hin zu einem gesellschaftlichen Beitrag. Dazu müssen die Individuen durch die Teilnahme an Ständen ihre „Standesehre“ gesichert haben und in dem Stand „anerkannt [...] sein“ (\$207): Nur mit dem Gefühl, geehrt und anerkannt zu 
Filipe Campello

sein, sind die Individuen auch in der Lage, einerseits ihre besondere Arbeit die Hegel „praktische Bildung“ (\$197) nennt - als einen gesellschaftliche Beitrag zu sehen und andererseits in diesen, universalisierten` Prozess seine Individualität auszudrücken.

Diese Artikulierung ist also weder gegen individuelle Befriedigung noch gegen die Interessen gerichtet, sondern besteht darin, in der Befriedigung, im Interesse selbst, dem Allgemeinen nicht zu widersprechen, und das ist nur durch die Bildung, durch den Übergang von der Willkür zum freien Willen möglich. Nur durch die hier durchgeführte Willensbildung lässt sich in der individuellen Befriedigung ein Ausdruck des Allgemeinen zeigen. Mit der Ausdifferenzierung, die in den Sphären der Arbeit und Korporationen stattfindet, nennt Hegel die „Natur der Besonderheit" als „wesentlichen Zweig der Gesellschaft“ (\$252). Insofern lässt sich bei kooperativen Verhältnissen diejenige Logik nennen, die Hegel als „Genossenschaft" bezeichnet, in der sich der „selbstsüchtige Zweck zugleich [...] als allgemeinen“ (\$251) versteht. Die Individuen sind aber auch in ihrem Interesse gesichert, so auch die "Sorge“ und „Bildung“ (\$252) um die Mitgliedern zu tragen, wobei die Genossenschaft und kooperativen Verhältnissen die Gestalt einer „zweiten Familie“ annimmt (\$252), welche die Individuen in ihrer selbstsüchtigen Tätigkeit zu einem allgemeinen Interesse hin bildet. 
Leidenschaften und Interessen: Hegel und die kritische Begründung der politischen Ökonomie

\section{Zwischen Teilnahmegefühl und institutionalisierter Solidarität}

Freilich ist es - besonders in Hinblick auf den Konsum - die Möglichkeit der Erfüllung individueller Präferenzen, die nicht als Sphäre ,gemeinsamer Interessen $\mathrm{zu}$ sehen ist, sondern die eine Struktur wechselseitiger Befriedigung ermöglicht ${ }^{23}$. Es kommt hier daher eine Spannung zwischen „Nachahmung“ und „Besonderheit“ (\$193): Wenn einerseits das Allgemeine so vorkommt, dass es sich nach den Anderen richtet, so etwa die „Art der Kleidung“ (\$192, Z.) und die Gewohnheiten, geht es andererseits beim Streben nach Besonderheit darum - wie wir bei der Rolle der Triebe und Leidenschaften gesehen haben - „sich durch eine Auszeichnung geltend zu machen“" (\$193). ${ }^{24}$ Diese ,Partikularitätstriebe hat Hegel auch als „Quelle der Vervielfältigung der Bedürfnisse“ (\$193) gesehen. Es ist aber durch diese Vervielfältigung - insofern dies „,keine Grenzen hat“ -, die auch extreme Formen wie "Luxus" (\$195) annehmen kann. Wenn einerseits also das hier betrachtete Interesse nicht mehr natürliche, sondern „gesellschaftliche Bedürfnisse“ als Medium für die Realisierung der Besonderheit darstellt, können sie andererseits zur „unendliche[n] Vermehrung der Abhängigkeit und Not“ (\$195) führen. Wie bei dem Beispiel des Luxuskonsums, nimmt sie ,abstrakte" und radikalisierte Formen auf die oben hingewiesene individuelle „Auszeichnung“ (\$193) an. So kann auf dem

\footnotetext{
${ }^{23}$ Vgl. Axel HONNETH 2011.

${ }^{24}$ Zur Diskussion vgl. FRANK 1999; VEBLEN 1949. In dieser Richtung erfasst schon Rousseau mit seinem Begriff von amour de soi einen grundlegenden Triebe der Selbsterhaltung, während der komparative Status eher die Struktur der amour-propre wiederspiegelt. Vgl. dazu NEUHOUSER 2008.
} 
Filipe Campello

Markt ein verfälschtes, nicht geformtes vernünftiges Interesse am Konsum entstehen: mit der Verhinderung von intersubjektivem Kooperationsgefühl und mit der Erfahrung wechselseitiger Befriedigung bilden sich die Akteuren verfälschte Vorstellungen davon, was für sie gut ist und was ihre Bedürfnisse sind, wie etwa bei Luxuskonsum, bis zur Annahme des Gelderwerbs als Ziel in sich selbst durch einem gewissen Prozess der Autonomisierung des Kapitals. In dieser Weise lässt sich nicht nur die Veränderung des Begriffes der Leidenschaft hin zu dem des Interesses interpretieren, sondern auch die durch den Kapitalismus beeinflusste selektive Veränderung der Gefühle, wobei nicht nur Kriterien der Kapitalakkumulation, sondern auch die von den Aktoren auf dem Market nachgefragten Konsumgüter, zur Gewohnheiten gewordene Präferenzen und Gefühle wie Liebe und damit verbundenen Praktiken durch kapitalistisch etablierte Kriterien vermittelt wurden. ${ }^{25}$

Weil in der bürgerlichen Gesellschaft einerseits die Individuen keine „natürlichen Erwerbsmittel“ haben (wie bei der Familie) und in der Moderne eine privilegierte Vorstellung von Ehre aufgehoben ist, muss sie andererseits diejenige Medien für die Subsistenz aller ihrer „Söhne“ behalten, die Hegel als Konsequenz des grundlegenden Schutzes der subjektiven Freiheit erfasst, einschließlich der entstandenen Armen; die bürgerliche Gesellschaft - schließt Hegel - „übernimmt die Stelle der Familie bei den Armen“ (\$241). Mit dem damit entstehende „Pöbet ${ }^{\star 26}$ (\$244) sind diejenige Gefühle, die sich als

\footnotetext{
${ }^{25} \mathrm{Zu}$ der umfassenden Diskussion des Verhältnisses zwischen Kapitalismus und Gefühle vgl. jüngst die Studien von Eva ILLOUZ (2003, 2007), die sich der Darstellung der Kommerzialisierung des Fühlens als kapitalistisches Konsumgut widmet.

${ }^{26}$ Vgl. zum Thema RUDA 2011.
} 


\section{Leidenschaften und Interessen: Hegel und die kritische Begründung der politischen Ökonomie}

konstitutiv für die Verhältnisse zwischen Subjekt und Sittlichkeit zeigen, blockiert, und daher spricht Hegel über die damit verursachten „Verluste des Gefühls des Rechts, der Rechtlichkeit und der Ehre, durch eigene Tätigkeit und Arbeit zu bestehen“ (\$244). Und in diesem Kontext sieht Hegel einige innerhalb der bürgerlichen Gesellschaft charakteristischen Gefühle, wie etwa die mit der Not verbundenen „Unfähigkeit der Empfindung und des Genusses der weiteren Freiheiten“ (\$243), oder das „Gefühl [...] ihrer Individuen von ihrer Selbständigkeit und Ehre“ (\$245), und - in anderen Sinn - über „Scham und Ehre, der subjektiven Basen der Gesellschaft“ $(\$ 245)^{27}$. Die in diesen sozialen Kontexten vorausgesetzten Gefühle werden damit blockiert, wodurch die Individuen ihre Tätigkeit nicht mehr ehrenhaft durchführen können.

Mit diesen Hinweisen lässt sich diejenige Gefühlsdimension erkennen, dessen normative Rolle Hegel für seine Theorie der bürgerlichen Gesellschaft verwendet und deren Scheitern ihrer konstitutive Rolle zwar vermeidet wird, sich aber mit anderen Gefühle wie „Empörung“ verbindet. So sieht man diese Dimension als einen Zusatz Hegels Textes, besonders in dem Grundbegriff der Gesinnung, die als Unterscheidungskriterium zwischen Armut und Pöbel charakterisiert. ${ }^{28}$

Aus diesen interpretativen Überlegungen lässt sich auch behaupten, dass es nach Hegels Prämissen auch sinnvoll wäre, eine auf den Markt und die

\footnotetext{
${ }^{27}$ Es ist erstaunlich, dass Hegel nur hier auf den Scham hinweißt, auch wenn eine ausführliche Diskussion über die Scham kohärent mit seiner Argumentation scheint.

${ }^{28}$ „Die Armut an sich macht keinen zum Pöbel: dieser wird erst bestimmt durch die mit der Armut sich verknüpfende Gesinnung, durch die innere Empörung gegen die Reichen, gegen die Gesellschaft, die Regierung usw.“ (\$244).
} 
Filipe Campello

Arbeit bezogene, dezentrierte Form von Interesse mit dem Begriff der Solidarität zu übersetzen; denn mit der Prämisse einer Verwirklichung des individuellen Willens als frei - durch die sittliche Dezentrierung - ergibt sich die Vorstellung, dass die Tätigkeit dem Anderen nicht nur Voraussetzung für die Befriedigung von Bedürfnissen und Selbstinteressen sind, sondern die Akteure fühlen sich tatsächlich motiviert und mitverantwortlich, sich für die Befriedigung der Bedürftigkeit zu engagieren. ${ }^{29}$ Hegel allerdings meint, dass die Armut sowohl durch subjektive Handlungen als auch durch einen institutionalisierten Mechanismus vermieden werden soll: Wenn zwar die

subjektive Hilfe ebenso in Rücksicht der besonderen Umstände als des Gemüts und der Liebe [erfordert], [...] [hängt] aber diese Hilfe für sich und in ihren Wirkungen von der Zufälligkeit abhängt, so geht das Streben der Gesellschaft dahin, in der Notdurft und ihrer Abhilfe das Allgemeine herauszufinden und zu veranstalten und jene Hilfe entbehrlicher zu machen. (\$242)

${ }^{29}$ Der von Axel Honneth in Anschluss an Durkheim entwickelte Solidaritätsbegriff zeigt alternativ ein nicht nur mit moralischen Gründen zu rechtfertigendes Prinzip (wie etwa bei Habermas), sondern weist tatsächliche Gefühle von Teilnahme und geteilte Zwecke als wichtige Basis für die Solidarität aus. Insofern sieht Honneth die Solidarität als eine "affektive Anteilnahme" (1994:210): "Beziehungen solcher Art sind ,solidarisch“ zu nennen, weil sie nicht nur passive Toleranz gegenüber, sondern affektive Anteilnahme an dem individuell Besonderen der anderen Person wecken" (210). Und auch in Anschluss an Hegels System der Sittlichkeit beschreibt Honneth die Solidarität mit der Formulierung eines „emotional aufgeklärten Anerkennungsverhältnis“ (45). Auch jüngst hat HONNETH (2011) den Begriff der Solidarität weiter entwickelt, in der das „Solidaritätsbewusstsein“ (327) als ein System von der Marktwirtschaft vorausgesetzten ,nichtkontraktuellen moralischen Regeln“ bezeichnet wird, das zur ,harmonischen Integration der wirtschaftlichen Einzelinteressen“ erforderlich ist (329). Dieser Gedanke, führt Honneth fort, „läßt sich in der von Hegel gewählten Sprache auch so ausdrücken, daß die vom Markt vorgenommene Koordinierung von bloß individuellen Nutzenerwägungen dann überhaupt nur gelingen kann, wenn die beteiligten Subjekte sich vorweg nicht nur rechtlich als Vertragspartner, sondern auch moralisch oder sittlich als Mitglieder eines kooperierenden Gemeinwesens anerkannt haben“" (329). Über Honneths Auffassung über den affektive Gehalt der Solidarität in der bürgerlichen Gesellschaft und denjenigen der Liebe vgl. IORIO;CAMPELLO 2013.

Revista de Filosofia Moderna e Contemporânea

Brasília, n² ano 1, 2013. 


\section{Leidenschaften und Interessen: Hegel und die kritische Begründung der}

politischen Ökonomie

Hegel weist insofern darauf hin, dass in dem Falle der Armut die subjektiven Handlungen nicht auszuschließen sind, aber wegen der Prekarität und Zufälligkeit, und um das individuelle „Gemüt“ und „Mildtätigkeit“ zu beschränken, wird ein institutionelles Gewerbe (eine „allgemeine Weise“) erfordert. ${ }^{30}$ Mit der realen Möglichkeit von Fehlern in diesem Prozess hat Hegel auch in Anschluss an die britischen Ökonomen die Notwendigkeit der Steuerung durch den Staat als notwendiges Medium betont - eine in gewisserweise ,institutionalisierte Solidarität ${ }^{6}$ - damit die vielschichtige Umfassung besonderer Interesse und damit eine gerechte Umverteilung realisiert werden könnte: Weil innerhalb der bürgerlichen Gesellschaft ein nicht zu überwindender Konflikt von Interessen stattfindet, sieht Hegel also die Wichtigkeit von „Abgaben“ (\$183, Z.), um die Fehler dieser Verhältnisse zwischen „Besonderheit und Allgemeinheit“ (\$184, Z.) überwinden zu können.

Hegels ,Realismus' besteht insofern darin, dass die Verhältnisse innerhalb der bürgerlichen Gesellschaft nicht nur in sich selbst organisch harmonisierend bleiben, sondern als immanente Spannungen charakterisiert sind. In diesen Sinn nennt Hegel die bürgerliche Gesellschaft „,das Schauspiel

${ }^{30}$ „Das Zufällige des Almosens, der Stiftungen, wie des Lampenbrennens bei Heiligenbildern usf., wird ergänzt durch öffentliche Armenanstalten, Krankenhäuser, Straßenbeleuchtung usw. Der Mildtätigkeit bleibt noch genug für sich zu tun übrig, und es ist eine falsche Ansicht, wenn sie der Besonderheit des Gemüts und der Zufälligkeit ihrer Gesinnung und Kenntnis diese Abhilfe der Not allein vorbehalten wissen will und sich durch die verpflichtenden allgemeinen Anordnungen und Gebote verletzt und gekränkt fühlt. Der öffentliche Zustand ist im Gegenteil für um so vollkommener zu achten, je weniger dem Individuum für sich nach seiner besonderen Meinung, in Vergleich mit dem, was auf allgemeine Weise veranstaltet ist, zu tun übrigbleibt" (\$242). 
Filipe Campello

ebenso der Ausschweifung, des Elends und des beiden gemeinschaftlichen physischen und sittlichen Verderbens dar“ (\$185). Es ist aber aus dem Grund, weil dieser Prozess an einer noch selbstsüchtigen Perspektive orientiert ist, dass hier die Stufe eines Bildungsprozesses notwendig ist ${ }^{31}$. Ohne diesen muss die schwierige Annahme einer aus der Verfolgung der individuellen Selbstbefriedigung resultierenden, harmonischen Befriedigung aller Teilnehmer aufgegeben werden.

Neben der Regulierungsrolle des Staates, was oft betont wird, besonders in Zuge von Marx' Kritik an Hegels Begriff der bürgerlichen Gesellschaft oder in dem keynesianischen Sinne einer kritischen Revision des Liberalismus, war für Hegel besonders wichtig, dass die der bürgerlichen Gesellschaft immanenten Strukturen die Beibehaltung der Selbstinteressen fördern: Nicht nur die staatliche Regulierung des Markt, sondern die dieser Sphäre immanenten Verhältnissen - wie Arbeit, Markt, und seine Institutionen wie die Korporationen - müssten diejenigen Bedingungen erfüllen, die die wechselseitige Befriedigung von den teilnehmenden Individuen ermöglichen. Es bleibt nach Hegel immer entscheidend, dass es nun um ein stets konfliktreiches Verhältnis vielfältiger subjektiver Befriedigungen geht. Die pluralistische Bedeutung, die für die Rolle der individuellen Autonomie wichtig ist, sollte aber den sozialen Verhältnissen nicht widersprechen. Insofern sind auch die Bildung individueller Interessen und Präferenzen - so verläuft Hegels Argument - stets von sozialen Kontexten untrennbar; denn nur innerhalb eines sozialen und konfliktreichen

${ }^{31}$ So sieht Hegel die hier schwierige Dialektik durchzuführen: „Meinen Zweck befördernd, befördere ich das Allgemeine, und dieses befördert wiederum meinen Zweck.“ (§184, Z.)

Revista de Filosofia Moderna e Contemporânea

Brasília, no 2, ano 1, 2013. 


\section{Leidenschaften und Interessen: Hegel und die kritische Begründung der politischen Ökonomie}

Charakters der bürgerlichen Gesellschaft kann der Markt entweder die wechselseitigen Bedürfnisse befriedigen, oder für seine begrenzte Lösung für die individuelle Befriedigung kritisiert werden. Hegel begreift aber immanent im Wirtschaftsprozess selbst das marktbezogene Interesse, die Arbeit und die Organisation der Stände und Korporationen als einen institutionellen Rahmen für die Durchführung des Lernprozesses; wenn das Individuum in diesen sittlichen Sphären die Befriedigung seiner ,Bedürfnisse' bzw. geformten Interessen sucht, wird es auf eine dezentrierte Perspektive orientiert, die für die Erfüllung seines Selbstinteresses unerlässlich ist. ${ }^{32}$ In dem Prozess sittlicher Handlungen lernt das Subjekt, dass nicht nur seine Interessen und Tätigkeiten, sondern die Tätigkeiten Anderer und der durch den Markt ermöglichte Tausch die Realisierung seiner Interessen ermöglichen.

Nach diesem Modell wird die Auffassung vom bereits Vorhandensein rationeller Akteure nach der ,Prämisse der Autonomie‘ für die Bildung individueller Interessen in Frage gestellt. Die Autonomie bildet vielmehr einen zu erreichenden Horizont, der - in Hinblick auf Hegels Begriff des freien Willens - von dem Inhalt des Interesses abhängt. Im Unterschied dazu weist dieses Muster darauf hin, dass die Interessen nicht nur in einem vernünftig kalkulierenden Prozess, sondern auch aus einer veränderten Form von Trieben und Begierden besteht, dessen konstitutive Kraft der Individualität untrennbar von seinem leidenschaftlichen Inhalt steht. Weil die Interessen

\footnotetext{
${ }^{32}$ Eine analoge, allerdings primäre und naturgeprägte Struktur haben wir bei der Formierung der Begierde gesehen, insofern die Beharrung des Bewusstseins auf seiner Partikularität und die Verneinung eines äußeren Allgemeinen, dialektisch ‘ zur Affirmierung und Anerkennung dieses Allgemeinen als konkreter Wirklichkeit führt.
} 
Filipe Campello

stets von einem leidenschaftlichen Inhalt und von sozial vermittelten Erwartungen, Normen und Anerkennungsverhältnissen abhängen, können sie weder als vernünftig noch als einseitig individuelle Interessen reduziert werden; denn in der individuellen Kalkulation des Interesses werden stets die sozial vermittelte Bestätigung und Abweichung von Präferenzen und ,Bedürfnissen` verbunden, wobei die auch mit dem Konsum verbundenen „Trieb nach Anerkennung “633 die Unmöglichkeit einer bloß vernünftigen Berechnung von Interessen zeigen, wobei eine vernünftige, instrumentelle Kalkulation nicht immer fähig ist, unter diffusen Bedürfnissen abzuwägen.

Hegel muss aber die Kriterien angeben, mit denen Arbeit und Markt kritisch evaluiert werden können. Es lässt sich allerdings ex negativo erkennen, das diese Sphären nur normativ legitimiert sind, wenn sie - nach dem vorher dargestellten Muster des freien Willens - die Selbst- und wechselseitige Anerkennung und individuelle Befriedigung einerseits und die an sittlichen Verhältnissen dezentrierte Interessen andererseits ausdrückt. Dass Hegel in der bürgerlichen Gesellschaft ein gespanntes Verhältnis zur Vorherrschaft der Realisierung selbstsüchtiger Zwecke erfasst, bedeutet auch, dass diese - als sittliche Sphäre - mit der Struktur und Verwirklichung des freien Willens innerhalb sozialer Verhältnisse verstanden wird. Für diese neue Stufe des Selbstausdrucks von Präferenzen und der Erfüllung von Selbstinteressen - der sich nicht mehr innerhalb der Familie befindenden primären Bedürfnisse entwickelt Hegel eine durch vielschichtige institutionelle Gewebe vermittelte Willensbildung, die Handlungsmuster und individuelle Zwecke als

\footnotetext{
${ }^{33}$ Vgl. dazu NEUHOUSER 2008.
} 
Leidenschaften und Interessen: Hegel und die kritische Begründung der politischen Ökonomie

einbeziehende Formen verstehen lassen. Die institutionellen Rahmen müssen die Garantien einer wechselseitigen Befriedigung fördern, die aber den am Markt und in der Arbeit handelnden Akteuren nicht extern bleibt, sondern sich auf ein legitimes Teilnahmegefühl gründet. 
Filipe Campello

\section{Literatur}

AVINERI, Shlomo (1972): Hegel's Theory of the Modern State. Cambridge: Cambridge University Press.

CHAMLEY, Paul (1963): Économie politique et philosophie chez Steuart et Hegel. Strasbourg: Dalloz.

CHAMLEY, Paul (1965): Les Origines de la pensée économique de Hegel. H. Bouvier.

COHEN, Jean L.; ARATO, Andrew (1999): Civil Society and Political Theory. Cambridge: MIT Press.

FRANK, Robert H. (1988): Passions within reason. The strategic role of the emotions. New York: Norton.

FRANK, Robert H. (1999): Luxury fever. Why money fail to satisfy in an era of excess. New York: Free Press.

GUNN, J. A. (1968): Interest Will Not Lie: A Seventeenth-Century Political Maxim. Journal of the History of Ideas, 29:4, 551-564.

HABERMAS, Jürgen (1966): Nachwort, In: Hegel, G.W.F, Politische Schriften. Frankfurt am Main: Suhrkamp.

HEGEL, G.W.F. (1970): Grundlinien der Rechtsphilosophie, Hrsg. von Eva Moldenhauer und Karl Markus Michel, Frankfurt a. M.: Suhrkamp

HERZOG, Lisa (2013): Inventing the market. Smith, Hegel, and political theory. Oxford: Oxford University Press.

HIRSCHMAN, Albert O. (1987): Leidenschaften und Interessen. Frankfurt am Main: Suhrkamp. 
Leidenschaften und Interessen: Hegel und die kritische Begründung der politischen Ökonomie

HONNETH, Axel (1994): Kampf um Anerkennung. Zur moralischen Grammatik sozialer Konflikte : Mit einem neuen Nachwort. Frankfurt am Main: Suhrkamp.

HONNETH, Axel (2001): Leiden an Unbestimmtheit. Eine Reaktualisierung der Hegelschen Rechtsphilosophie. Stuttgart: Philipp Reclam.

HONNETH, Axel (2011): Das Recht der Freiheit. Grundriß einer demokratischen Sittlichkeit. Berlin: Suhrkamp.

HORSTMANN, Rolf-Peter (1997): Hegels Theorie der bürgerlichen Gesellschaft, In: Siep, Ludwig (Hg.), G.W.F. Hegel. Grundlinien der Philosophie des Rechts. Berlin: Akademie Verlag. 193-216.

ILLOUZ, Eva (2003): Der Konsum der Romantik. Liebe und die kulturellen Widersprüche des Kapitalismus. Frankfurt am Main / New York: CampusVerl.

ILLOUZ, Eva (2007): Gefüble in Zeiten des Kapitalismus. Frankfurter AdornoVorlesungen 2004. Frankfurt am Main: Suhrkamp.

IORIO, Gennaro; Campello, Filipe (2013): Love, Society and Agape: An Interview with Axel Honneth. European Journal of Social Theory, 16(2) 246258.

NEUHOUSER, Frederick (2008): Rousseau's theodicy of self-love. Evil, rationality, and the drive for recognition. [Oxford]: Oxford University Press.

PELCZYNSKI, Z. A. (1984): The State and civil society. Studies in Hegel's political philosophy. Cambridge [Cambridgeshire] / New York: Cambridge University Press. 
RIEDEL, Manfred (Hg.) (1975): Materialien zu Hegels Rechtsphilosophie. Frankfurt: Suhrkamp.

RUDA, Frank (2011): Hegels Pöbel. Eine Untersuchung der "Grundlinien der Philosophie des Rechts". Konstanz: Konstanz Univ. Press.

VEBLEN, Throstein (1949): La teoria della classe agiata. Torino: Einaudi.

WALTON, A. S. Economy, utility and community in Hegel's theory of civil society. In: Pelczynski, Z. A.: The State and civil society. Studies in Hegel's political philosophy. Cambridge [Cambridgeshire] / New York: Cambridge University Press. 